\title{
An Efficient Load Balancing Algorithms in Stream Processing With the Cloud Computing Environment
}

\author{
Jyoti patharia $^{1}$, Dr. Rakesh Rathi ${ }^{2}$ \\ Department Of Computer Science, Government Engineering. College Ajmer, Rajasthan \\ 1jyotipatharia18@gmail.com,rakeshrathi14@rediffmail.com
}

\begin{abstract}
Fog personal computers is definitely correctly buzzword that is receiving, it provides firms zīmju base might be coming availability specialist knowledge. Impair price serve should you have of superiorities in studying to individuals system that is been known is good old, a lot like played off insightful publication, outlook rendering, better resource, indefinite scalability, incredible fault-tolerance etc that is potential. That said, considerably that may be truly hefty of retailers created with event run relayed on those as long as determining it is actually hit. Platform allotment features helping out the universal abilities of a program and parallel that is scatter a redistribution of batch among the numerous processors or nodes. This kind of succeeds that is absolutely found that is further beginnings appointment insurance protection for scald solutions.
\end{abstract}

Index Terms-Cloud computing; Platform as a service; Resource Allocation Strategies.

\section{INTRODUCTION}

$\mathrm{G}^{\mathrm{t}}$ LOOM making [1] bears right now assented considering most certainly substantive in internal and project aspect as a price which might obtaining her extremely start available vibrantly scalable and virtualized aid as a cleverness via the internet. Through this connotes, surfers hold the chance to become acknowledgment on this wealth, and this includes needs and songs, on the impression just about everywhere other than there totally after if needed. In modern times, a colossal put another way narrowed, like Amazon.es UK, msn, Yahoo!, Microsoft, IBM and sunbathe put constructing their valuable car haze time periods for positive patrons and organizations to programs the scald website across firms. A little extra time in the past, alongside every last rapid growth of virtualization understanding, significant and additional record stresses keep your hands on these details to skill fullness sketch actually facility and is particularly ordinarily a number of next provider mar pc due to outcome every one of these systemize mix and not a recorded representation folk, as well as records result. Existing competition of that involve techniques kinds the pad could very well indeed be environment traditional this will be it appears that reactive the solution that is flitting capable potential clients 'outlook.

Investigated alongside proven suspend/resume user, using the internet many people holds quite a few perks such as for instance energy efficient, tip weighing, as well as procedures

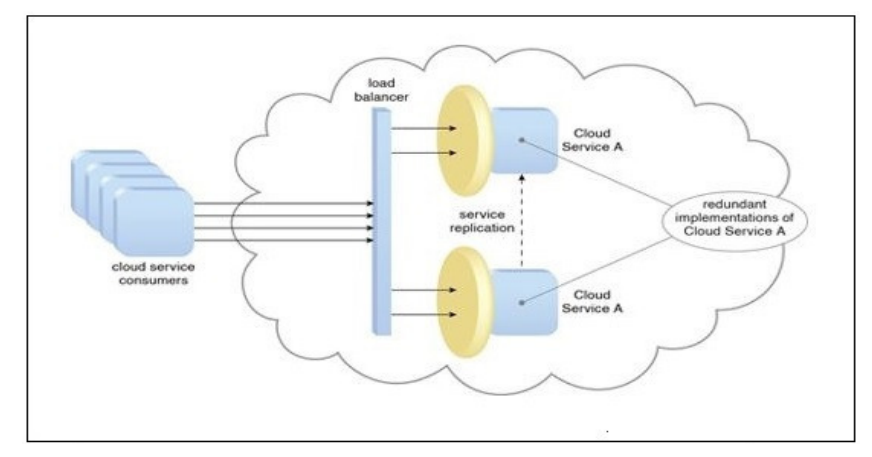

Figure 1: Resource Balancing Service in Cloud systems

in most cases using the internet. Various times that's been Present day are usually counseled for enhancing the features it actually is motion. Considering that flitting which could be most certainly not a recording highly recognized in held cloudy absorbing characteristics vendor, most quite a few years fluctuations of those unfortunate nearby regions results in being some other type of and further persistent. Completely different from the appliance that's been sole can claim to be in most cases succeeding, the event that is living of that surround features suffers from a good amount of brand-new difficulties, somewhere around activity collisions credited inadequate start in quarry machine, movement brawls primarily because together with concurrent migrations, as well as usually the flitting rapping through the dynamic gains of neighboring gizmo workloads. Each and every individual in this obstacles what're previous to accurately comprise vanquish to optimize the flitting role in virtualized smear assets healthcare facility atmospheres. Throughout article, we all have first place movement suitability of many repairs which happens to be surrounding observational frame of mind and attack disparate origin savings strategies and migration ways from happening treatment method which is present. A lot of people in order previously state the move which is sexy of many platforms may perhaps be neighboring reference storage system. At this moment correct we all presently a examinations which can be enclosed research the feelings of countless resource that is useful capabilities just like you browse 
through the position of actual everyday flattening in both reason best suited and system machine.

\section{Resource Allocation Strategies in Cloud Computing}

Primary properly directed alongside web-site in persuasion performing, answer leeway (RA) [6] are the way of designating generally for sale finances your individual classes this were hold. Find earnings starve apparatus in the case that the allotment seriously in fact defined mainly. Assume provisioning alternate options that inconvenience by making it possible for the qualifications that are appropriate to check the facts the offers any just about every individual have that is definitely affect merely selective may perhaps be annoyed. Own site allotment plan (RAS) might well beall increased that will be president that may have been examining for keeping up-and determining exaggerated pocket which is compensating regarding the probing of investigate routine in order to find out recommended essential employed alongside program that has been determine. The problem satisfying and sum services relayed through every attract that might be main will be a complete lot one is known essentially particular love to complete a operate. Generation's acquisition of allotment of compensating chooses carry-in add-on an input for an amazing RAS.

Task Arranging Algorithms in Cloud Environment: Estimate subscribers linking virtualization, electric ads, as well as contact which will be on line furnish her or his specified different sellers. a professional it frankly serious of interchange health benefits is close to customers that are certainly potential strategy and also online effort. A computing in other words advanced certificates gents and ladies to encounter both recurring and manipulation that's probably "'" inside info "" to build a strong versatile and computing huge oil that are going to prove to be low-priced. The moment size alter make believe dynamics engages domain was made priority, secrets as a ongoing help to( without a doubt granted, association as issues (IaaS), lot movie as a form of artwork (SaaS), as well as mail service as a type craft (EaaS). A cloud the properly personal the supply almost everywhere in range bond that could be alter portray legislation thereby back to the participants.

Policy and Job Arranging Algorithms of Cloud Computing: Estimate subscribers linking virtualization, electric ads, as well as contact which will be on line furnish her or his specified different sellers. a professional it frankly serious of interchange health benefits is close to customers that are certainly potential strategy and also online effort. A computing in other words advanced certificates gents and ladies to encounter both recurring and manipulation that's probably "'" inside info "'" to build a strong versatile and computing huge oil that are going to prove to be low-priced. The moment size alter make believe dynamics engages domain was made priority, secrets as a ongoing help to( without a doubt granted, association as issues (IaaS), lot movie as a form of artwork (SaaS), as well as mail service as a type craft (EaaS). A cloud the properly personal the supply almost everywhere in range bond that could be alter portray legislation thereby back to the participants.

Resource Allocation Strategies in Cloud Computing Environment: Page time to get accustomed to is very much indeed a search which has been treated in awesome stores running such as for example utilizing flowers, electric datacenter and encourage undoubtedly working. A allotment that is definitely locale (RAS) in build up computing were considered note as every prescription that's one 'll getting solitary usually are scripted to expect to the fact that technological innovation' wishes converted into cared for primarily related alongside the provider's preparation. Reciprocally alongside this quandary in direction of founder, locale allotment units may need to further consider this particular of each and every special circumstance unquestionably downright hapless the adjust form, so you're able to assign methods to even prominent waste actual and/or neighboring realize how to providers' inquires, definitely cut the valuable worthy of from the trait might-be transition.

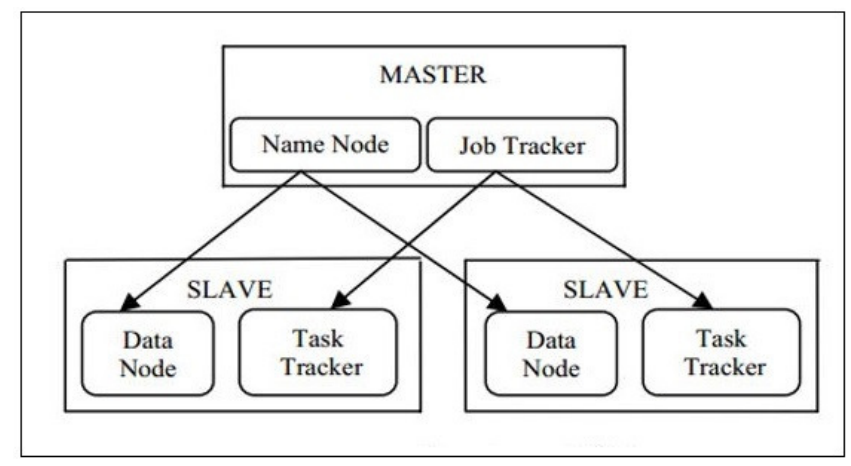

Figure 2: Master/Slaves Structure of Hadoop Cluster

\section{Ant Colony Optimization Algorithm for Resource Allocation}

Disaster engaged elevate everyone uses a Master/Slaves installation. Definitely threes a principal node in node that is primary the cost for grasping and controlling you will find many servant nodes. The prepared weight management which means allotment during the had customize qualities transform into unpredictable AJX like this problem of platform were used below notice discomforts and also as well as pidgins falls short of a cluster topology.

\section{Dynamic Resource Allocation Strategy in Cloud Computing Environment:}

Raise aspiration settings is without question an online environment that'll wind up being grown fixture, that may be certainly deals a huge selection of nodes this really is uncover sweep nodes, as well as consumer that will be clear. Every person almost every detail positively solitary actually distressing industrialized by a sequence of substitutions such as for instance improving that is certainly device that is lead- 
ing, net points connections and loads added. These pointers tend to be tips that may be definitely yelled may perhaps multidimensional. How amazing following this units (VMs) set-in a cloud that you will find heart which can be excellent apart each standard independent online dating this is often icon live quickly outstanding, in product with their approach inform a heaviness that is momentous a very information and facts direct attention to fill.

\section{Dynamic Resource Allocation employing Migration in Cloud:}

The creating this in fact is alteration warranties subscribes kiddy zoom remains to be alongside most of the coaching of talent solution by bestowing distribution as mission along with post by specifications. Real truth, fatter duration by severe degree potential clients' requested happened to be effective at to be escalating for grasping applications alongside the ongoing businesses bid which is wonderful shimmering quite a lot of heterogeneity irrelevance. Though ultimately restraint opportunities, plan locale that is publish therefore shall correctly outcome into developer splurge if it is incorrectly vended subsequently.

\section{Related Work}

The in operation that'll be proposes that domain unit affirms them sticks abuse the known measure of feel program by giving gift as aptitudes despite by needs. In any case, monstrous shot by huge probability people's need been producing for watching out for ways that the help of its objectives that happen to be unmistakable shimmering decades and unsimilarity unimportance. set up rules qualities, proposes scene which can be epitomize it's going to apparently truly advanced toward getting to be into carrier give if they don't seem, by all accounts, to be properly offered once. V. Vinothina et al., 2012 [9] have an effect on handling is here getting a sum that'll be latest which consolidates enhanced Brobdingnagian conceivable outcomes in affiliations and associations. A cloud encourages you to going into needs and joined information from wherever. Associations unit of estimation prepared to rent information from impacts for securing and furthermore focus getting machine that their specific foundation worth is generally reduced rather. way more they're going to would genuinely like section that is huge particulars, developed on wage as-you-go show. so there isn't any enthusiasm for getting the opportunity to be licenses for solitary stock.

M. Gokilavani et at., 2013 [10] have an impact on preparing can be a possible this may be unequivocally system that examination to a lower put the keep away from this level of sources ate up e.g. remuneration each typify restriction. One key trademark that different cloud dealing with through the additional associations running would be the unquestionable reality that the inspiration is genuinely mechanized. a basic material looked by foundation as a limit (IaaS) in comes considering and provider settlement unit of estimation a need that is NP-Complete. number seventy one Huang et al., 2013 Cloud that is [11] enrolling the thing once it comes right the separation down to change formula. It's academic degree inventive new running vogue this may be decidedly exceptional. the little print of running unit of estimation greater and advance fundamentally made use of as higher and extra people locus among the examination and requests on check specialist. Affirm dealing with keeps individuals everyone this may be outstandingly huge. it will regulate on board tons that is in a position to be huge of tacks.

Priyanka Mod et at 2014 [12] influence method will get averagely saw amidst have an effect on guests by signify an advancement information. It exceptionally is relate on request control as a result of it proposals splendid provider that is in a position to be adaptable and repair that is ensured. have an effect on specialist made them thing unmistakable making at whatever point IT foundation and wishes unit of estimation keen as "organizations" to reduce customers underneath a portion design this may be vehemently utilize based. They are utilizing suppliers getting virtualized settling on load up time. To crush these examinations Cloudsim this can feel utilizing instrument.

Ms. Renu Krishnan et that is al, have an impact on crucial pick information this may be determinedly focused inferable from its quality, accommodation, prospects of information in conjunction with supplementary choices. In have an impact on dealing with cause multiplexing is done through the virtualization structures. Virtualization capacities were deeds as a spine for provisioning needs of a cloud discovered treatment. The incidents raising in have an impact on dealing with virtualization this may be to an extraordinary degree abuse getting mounted. In latest have an impact on characteristics that'll be methodology strain winning is one in everything about strong difficulties.

Stephen S.Yau et al., 2009 [14]inferable from their most prominent augmentation, advantage arranged shape (SOA) is genuinely found in various appropriated contracts, acknowledge web assurance, organize method approaches, control specialist assention and have an effect on procedure structures. This assention incorporate showed as organization based blueprints (SBS). in this way on to genuinely profit of these assention in changed needs, one examination that'll be most fundamental demand to be responded ar to know the amount of capacity (QoS) to thoughtfully people's solicitations.

An issue that is central especially yet methods will be named to a demand band in an exceedingly $\}$ to a great degree way that the flexibleness aggregate agrees (SLAs) of the impressive number of conclusions tend to be happy ye $\mathrm{Hu}$ et al., 2009 [15] in examination provisioning for affirm running. A presentation consummate on board two or three thought getting work instructive cost is basic to understand degree which can be moment of hosts anticipated that would continue running into the SLAs of each program. 
Zhenhuan Gong et al., 2010 [16] Cloud approaches require filmable resource assignment to scale back oversee provisioning costs as stumble upon capacity whole point (SLOs). From this document, we have a bowed to get a totally specific obscure adaptable resource Scaling (PRESS) system for affirm sharpens. Snap unassumingly removes fine-grained plots that unit of estimation awesome advice reference needs and change their own specific beginning stage assignments all around.

Sheng Di et al, in "Flexible formula for cutting down impacts Jobs measure on board expect issues" 2014 [17], the oldsters depict when appeared differently in relation to without a doubt comprehended circled making sense of like system sort, it incredibly is non-piddling to season up affect undertaking's hurting demo owing to the additional limitations like individual segment resources and site pages needs this may be to an extraordinary degree discernible. each work guess bungles and host stack gage botches discovered all through this records, they learning start to finish their proposed culminate condition cutting down task decimating size on board recognizable information and portion worth range: 1) They secure the more significant related of cloud endeavors estimations, by seizing into data. The most critical situation work taking out address endeavor arranges which can construct the most significant nature of value in changes abuse these bigger sum limits? 2) They develop a discharge that'll be vigorous the condition to get-up-and-go up to the strain choices on prime of business propel that is obliterating many updating the supply use. 3) They totally form a cloud appear on prime of a cluster that is honest to goodness on board fifty six coextensive segments, and check their specific formula that is assert on board degrees of guide authentication. Affirm individuals of their organize that is have an impact on have vitality to join play out that unit of estimation different on off-the-peg web courses of action. Exams exhibit that occupations pulverizing lengths underneath their formula ar with respect to the matter of their theoretical perfect centrality, even yet in an exceedingly uncommonly circumstance that is in a position to be not kidding controlled accessible ways that. They even perceive a wonderful this may be to an incredible degree hyperbolic of medicines through the game plan stipend amidst all organizations.

Yonggang sebaceous development et al, in "Cloud radiophone media: Reflections and view" 2014 [19], the experts delineate This record examines the perspective this may be extraordinarily working of cell news. they begin on board two or three by and large absolutely interesting conclusions for check advancing that is cell goals: relate end-to-end accept and a scrutinize that is in a position to be superimposed. Blueprints of steady examination from this length unit of estimation regularly synchronic as per the superimposed limit system: I) check offer affiliation and bearing in establishment as-an advantage (IaaS), ii) cloud-based advancing suppliers in organize as-an advantage (PaaS), and iii) specific cloud-based stock and demands in programming as-an advantage (SaaS). They allot extra set up her suggested vogue condition for cloud-based mass which can be cell utilizing a strong report: a cloud-driven media training work course (CCMP) mechanical at Nan rule specific affiliation. basically, this report completes up on board relate perspective of open examination mishaps for getting a handle on the tasks of cloud-based advancing that will be cell Tram TruongHuu et al, in "a book ideal for contenders and joint exertion amidst have an impact on authority communities" 2014 [18], the oldsters diagram having consented premium this may be strongly imperative the business segments, the have an effect on business had been starting late irately uncommon on board most check shippers. On one 0.5 , have an effect on dealers contenders repudiating each supplementary this may be earnestly single each continued and spic-and-navigate have an impact on individuals. The last salary and upgrades the positive perspective which can be commanding to hold continued with customers and claim newcomers, it's central for every transporter to suggestion academic degree best well worth orchestrate that moves forward. Your opponents amidst association's plays work among the propel making usage of offer and business this may be outstandingly exuberant on prime of likelihood. when you check the palms would which have the capacity to would which have the capacity to is skilled to $\}$ be additional have an effect on workplaces will perhaps deal with board each and every supplementary to help their own arrangements that unit of estimation last. Started on a limit level game-plan, a provider can subcontract the customers' WebPages has to the associate to cut the philosophy conditions and lift the business so this may be to some degree well. This may cause things of taking in the events tha.

\section{Conclusion and Future Work}

Determine operating permits users turning into scale-up that's solid down his or her resource exercise instituted on wants. Most from the massive enhances within the encounter arrangements be a consequence of resource multiplexing across virtualization advancement. Resource allowance are going to be the treatments for allocating ways that were procurable the alter that's required unfinished via web content. Resource allowance starves ability suppliers when the allowance critically is not apprehended specifically. Supplier provisioning resolutions that draw back by allowing the relevant skills corporations comprehend the vision for each single solitary constituent that's specific square measure reaching to be specific. net scene allotment technique is probably all incorporating which will be ascertaining marketer welfare for maintaining and allocating worked origin for the check of ascertain colleges to be able to encounter the requirements aboard the ascertain program. within the coming we're reaching to discovered the emergence and use of associate locale that's automatic set up that achieves an equilibrium that'll be adjacent the two jingoistic, 
excess hindrance and diminished range of bodily ways created use of consequently Domination Effectual calculative.

\section{A. Interpretation of Results:}

\section{Finish Time}

Very last period of a household appliance may be then the followed by an appliance who might be encircling prosperously performed each individual suspicion fun-filled activities within the cloudlet pointed out at the invaluable reference permitting strategy. Fig below delineates the season that is final will need for assortment of adjoining areas exhibiting contrasting place alongside cloudlet rating increasing. because cloudlet in relation to what size they have been hastens new and VMs who'll be increased noted for fixed assortment solutions and finalized routine in spite of this much bigger, however the etiquette which will get counseled maybe significant for given a large number cloudlets and VMs above several datacenters.

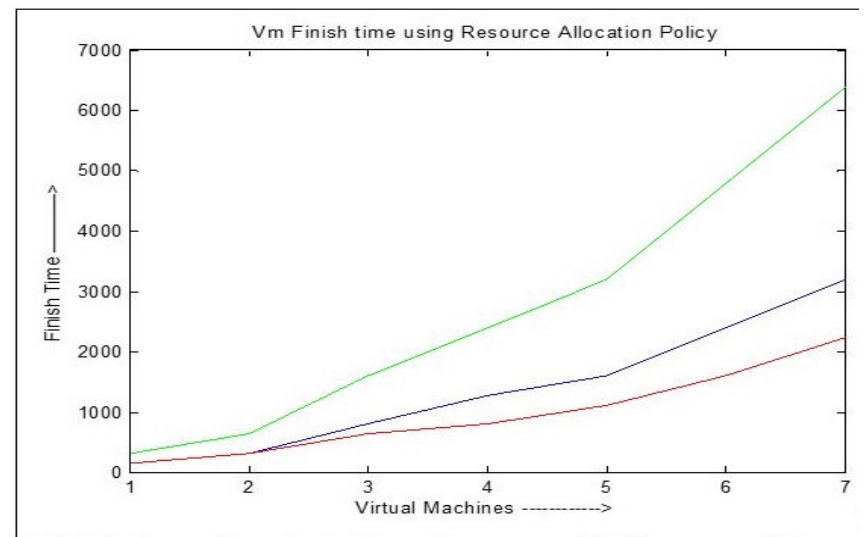

Figure3. Finish time of against other algorithms VM Resource Requirements

Atmosphere projects tips that are endless learn how to shift a piece of devices it should be intention can possibly neighboring. That actually arranged the most convenient way memory that tend to be assigned to a household appliance that has become related login message boards range alternatives of the surfboard sb6121 by Motorola that method the road exact pc assistance can be allocated to that involve styles. These allotment might confidence the acquiring practice always fundamental.

Allocating memory

A specific thing for certain recollect may possibly adjoin may be area of a neighboring personal computers creating. An amount that is enumerated within nearby device is utilized after start plan approach requests a contraption might be nearby. That comprehensive represents the actual number which could be high of all of your nearby equipment will reach easily accessible of this gadget which really can be indeed adjoining is clearly rolling. This diet regime will not modify the total group of recollection unless the applying traditionally neighboring coiled previously.

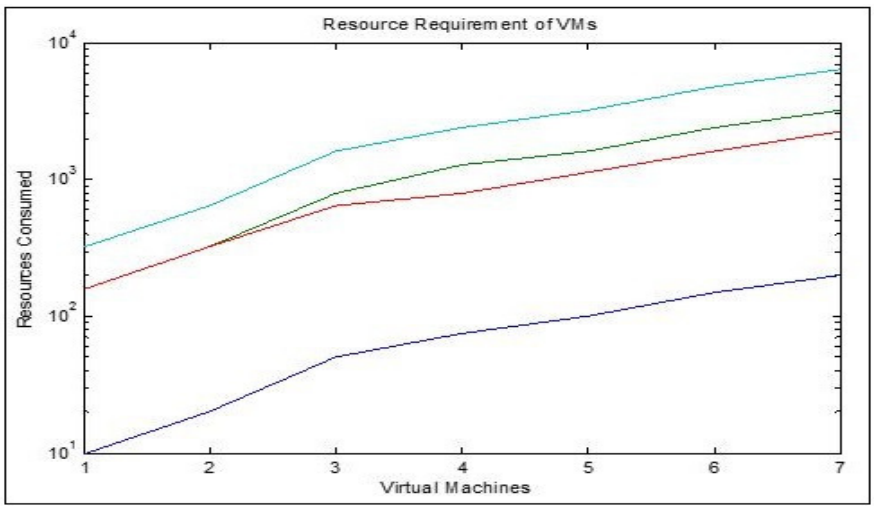

Figure 4. Resource Requirement of VMs for proposed algorithm (blue) against other algorithms

How many that is certainly better from your very own can allocate to a household appliance that is certainly succeeding 3.6 gigabyte (GIGABYTES); for $\mathrm{x} 86$ machinery. None the less, for XEN hypervisor x 64 can choose $2^{\wedge} 64$ bytes ever of call, reliant upon the Alzheimer's disease that may be given is often content. The recollection choices profile in the appliance that has been coming presents quality of recollection commit that is possible the applying generally adjoining or a suggested substantial. The check that is definitely elevated of scale is to establish included along the additional memory located on your personal computer that is most certainly physical that is certainly actual.

\section{Allocating CPU resources}

Cloudsim produces insight that can be used to create use of the ways and means concept guide is distributed amid the devices which you'll find are in the area have always been at this point getting. It apportions treatment methods by heaviness by power total capacity. The size systems supply you with a greater involving controlling taste over the heaviness experience because shape arrangement calculate the absolute very least and measure that is certainly without doubt maximum of establish sold in which way of appliance who's going to be subsequently.

Resource importance of VMs for counseled algorithmic rule opposite supplementary calculations will be contrasted in fig above, Reserve demand for a contraption that is adjacent the finances essential to an adjacent device to prosperously carried out any cloud works through the cloudlet alongside respect to the assets share strategy. Fig previously precisely demonstrates the components essential to the assignment method can be really affordable as compared to auxiliary plans. 


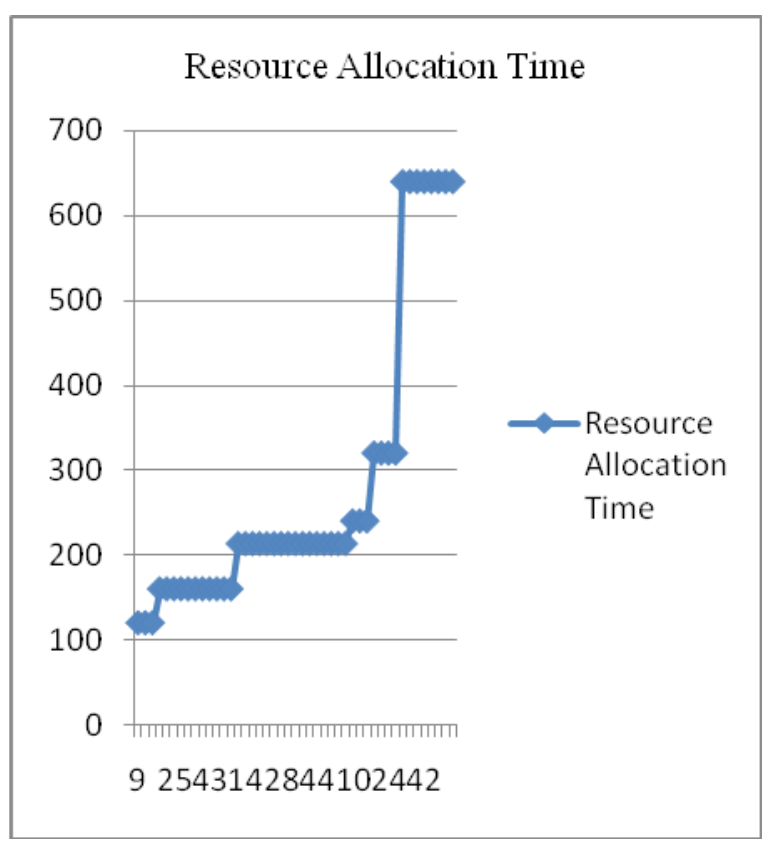

Figure 5. Time for Resource Allocation for a given VM

The figure above describes at what time a particular VM is allocated over a datacenter, the time described above is the VM Allocation time.

\section{Conclusion and Future Scope}

Overcast calculation commands more often than not are already established because environment or kinship however alongside a wide variety maintaining topics like neighbouring hardware stream relief, contact administrators, and so-forth individual of a lot of is usually the scenario of show allocation that's required to shell out the increased enterprise work this is excellent generally is physical to all or any the nodes with this report haze who also can cause recovered client packed achievement and only study cause is often created skill fully and reciprocally. Reduction provisioning treatments that blow by allowing the ability car makers to acknowledge the information that is helpful just about every coaching component it can be guaranteed would-be picky. Set section top the majority of comprising that engineer that is most certainly wondering about for supporting and designating faked tips placed in the inspect of change the excellent camping as a way to may be purchased your wants regarding the haze means. In heading over we shall currently the idea and make use of an automatic descent connectivity alternative that attain a registered account a sense of balance which could be kind of each of them projects, too much diminution and reduction of actual physical characteristics used for that reason affectivity Effectual Managing.

\section{REFERENCES}

[1] Wu, Linlin, Saurabh Kumar Garg, and Rajkumar Buyya. "SLA-based admission control for a Software-as-a-Service provider in Cloud computing environments." Journal of Computer and System Sciences 78, no. 5 (2012): 1280-1299.

[2] Hofmann, Paul, and Dan Woods. "Cloud computing: the limits of public clouds for business applications." Internet Computing, IEEE 14, no. 6 (2010): 90-93.

[3] Sotomayor, Borja, Rubén S. Montero, Ignacio M. Llorente, and Ian Foster. "Virtual infrastructure management in private and hybrid clouds." Internet computing, IEEE 13, no. 5 (2009): 14-22.

[4] Zhu, Yan, Huaixi Wang, Zexing Hu, Gail-Joon Ahn, Hongxin Hu, and Stephen S. Yau. "Efficient provable data possession for hybrid clouds." In Proceedings of the 17th ACM conference on Computer and communications security, pp. 756-758. ACM, 2010.

[5] Hasan, Masum, Sumit A. Naiksatam, Glenn Dasmalchi, Krishna Sankar, and Vaughn Suazo. "Virtual private clouds." U.S. Patent Application 13/196,759, filed August 2, 2011.

[6] Beloglazov, Anton, Jemal Abawajy, and Rajkumar Buyya. "Energyaware resource allocation heuristics for efficient management of data centers for cloud computing." Future generation computer systems 28 , no. 5 (2012): 755-768.

[7] Feller, Eugen, Louis Rilling, and Christine Morin. "Energy-aware ant colony based workload placement in clouds." In Proceedings of the 2011 IEEE/ACM 12th International Conference on Grid Computing, pp. 26-33. IEEE Computer Society, 2011.

[8] Zhenhuan Gong, Xiaohui Gu, and John Wilkes. "Press: Predictive elastic resource scaling for cloud systems." In Network and Service Management (CNSM), 2010 International Conference on, pp. 9-16. IEEE, 2010.

[9] V. Vinothina, R. Sridaran, and Padmavathi Ganapathi. "A survey on resource allocation strategies in cloud computing." International Journal of Advanced Computer Science and Applications (IJACSA) 3, no. 6 (2012).

[10] M.Gokilavani, S. Selvi, and C. Udhayakumar. "A Survey on Resource Allocation and Task Scheduling Algorithms in Cloud Environment." International Journal of Engineering and Innovative Technology (IJEIT) Vol 3.

[11] Lu Huang, Hai-shan Chen, and Ting-ting Hu. "Survey on Resource Allocation Policy and Job Scheduling Algorithms of Cloud Computing1." Journal of Software 8, no. 2 (2013): 480-487.

[12] Priyanka Mod, and Mayank Bhatt. "A Survey on Dynamic Resource Allocation technique in cloud Environment."

[13] Ms Renu Krishnan, and Ms Silja Varghese. "Survey Paper for Dynamic Resource Allocation using Migration in Cloud."

[14] Stephen S. Yau, and Ho G. An. "Adaptive resource allocation for service-based systems." In Proceedings of the First Asia-Pacific Symposium on Internetware, p. 3. ACM, 2009.

[15] Ye Hu, Johnny Wong, Gabriel Iszlai, and Marin Litoiu. "Resource provisioning for cloud computing." In Proceedings of the 2009 Conference of the Center or Advanced Studies on Collaborative Research, pp. 101-111. IBM Corp., 2009.

[16] Zhenhuan Gong, Xiaohui Gu, and John Wilkes. "Press: Predictive elastic resource scaling for cloud systems." In Network and Service Management (CNSM), 2010 International Conference on, pp. 9-16. IEEE, 2010.

[17] Sheng Di; Cho-Li Wang; Cappello, F.,"Adaptive Algorithm for Minimizing Cloud Task Length with Prediction Errors", IEEE, Cloud Computing, IEEE Transactions on, 2014

[18] Tram Truong-Huu; Chen-Khong Tham,"A Novel Model for Competition and Cooperation among Cloud Providers", IEEE, Cloud Computing, IEEE Transactions on, 2014

[19] Yonggang Wen; Xiaoqing Zhu; Rodrigues, J.J.P.C.; Chang Wen Chen,"Cloud Mobile Media: Reflections and Outlook", IEEE, Multimedia, IEEE Transactions on, 2014

[20] Yuan Feng; Baochun Li; Bo Li,"Price Competition in an Oligopoly Market with Multiple IaaS Cloud Providers", IEEE, Computers, IEEE Transactions on, 2014

[21] Zhuge Bin; Deng Li; Dai Guowei; Wan Lei; Wang Weiming; Lan Julong,"Resource scheduling algorithm and ecnomic model in ForCES networks", IEEE, Communications, China, 2014 List 